\title{
Extração e análise de propriedades físico-químicas do óleo de açaí (Euterpe oleracea
}

\section{Mart.)}

\author{
Extraction and analysis of physical and chemical properties of açaí oil (Euterpe oleracea Mart.)
}

Extracción y análisis de las propiedades físicas y químicas del aceite de açaí (Euterpe oleracea

Mart.)

Recebido: 19/06/2021 | Revisado: 25/06/2021 | Aceito: 26/06/2021 | Publicado: 11/07/2021

\author{
Rafael Costa de Castro \\ ORCID: https://orcid.org/0000-0002-1294-5197 \\ Universidade Paulista, Brasil \\ E-mail: rafaelcastroflor2@gmail.com \\ Luan Felipe Santana Costa \\ ORCID: https://orcid.org/0000-0003-0601-9979 \\ Universidade de Passo Fundo, Brasil \\ E-mail: luanfelipesc0@gmail.com \\ Lorena Marina Dias de Alcântara da Costa \\ ORCID: https://orcid.org/0000-0003-0978-7578 \\ Universidade de Passo Fundo, Brasil \\ Guilherme Bandeira Candido Martins \\ ORCID: https://orcid.org/0000-0002-1387-9355 \\ Universidade de Brasília, Brasil \\ E-mail: bandeira007@gmail.com
}

\begin{abstract}
Resumo
O açaizeiro (Euterpe oleracea Mart.) é uma palmeira nativa da Amazônia, que ocorre em grandes extensões no estuário amazônico. Durante o processo de conservação e extração de óleos, fatores como umidade, temperatura, enzimas lipídicas e presença de luz, podem afetar o perfil lipídico, favorecendo a hidrólise e liberando ácidos graxos. A presença de ácidos graxos influencia na qualidade dos óleos. Este trabalho teve como objetivo a extração do óleo de açaí por solvente orgânico e a análise de alguns parâmetros físico-químicos como: densidade, níveis de acidez, e perfil glicídico. Para a extração foram utilizados o aparelho soxhlet e o hexano como solvente. A extração do óleo de açaí não apresentou bom rendimento, devido à escolha de se utilizar os frutos inteiros que tem grandes quantidades de fibras envoltas nas sementes. A densidade foi analisada através do densímetro 35n da ANTON PAAR, a acidez foi medida através de titulação por $\mathrm{KOH}$ e para o perfil glicídico o óleo foi submetido a análise de espectroscopia no infravermelho por transformada de Fourier (FTIR), utilizando o aparelho IR Prestige-21 da Shimadzu. A densidade apresentou-se um pouco baixa. O nível de acidez apresentou valores um pouco elevados, indicando a formação de ácidos graxos, no perfil glicerídeo foi possível observar a presença de ácidos graxos livres, o que justifica a acidez.
\end{abstract}

Palavras-chave: Açaí; Euterpe oleracea Mart; Óleo de açaí.

\begin{abstract}
The açaí palm (Euterpe oleracea Mart.) is a palm tree native to the Amazon, which occurs in large extensions in the Amazon estuary. During the process of conservation and oil extraction, factors such as humidity, temperature, lipid enzymes and the presence of light can affect the lipid profile, favoring hydrolysis and releasing fatty acids. The presence of fatty acids influences the quality of oils. This work had as objective the extraction of açaí oil by organic solvent and the analysis of some physical-chemical parameters such as: density, acidity levels, and glycidic profile. For the extraction, soxhlet apparatus and hexane as solvent were used. The extraction of açaí oil did not show good yield, due to the choice of using whole fruits that have large amounts of fibers wrapped in the seeds. Density was analyzed using an ANTON PAAR 35n densimeter, acidity was measured by $\mathrm{KOH}$ titration and for the glycidic profile, the oil was submitted to Fourier Transform Infrared Spectroscopy (FTIR) analysis using the IR Prestige- 21 of Shimadzu. Density was a little low. The acidity level showed slightly high values, indicating the formation of fatty acids, in the glyceride profile it was possible to observe the presence of free fatty acids, which justifies the high acidity.
\end{abstract}

Keywords: Açaí; Euterpe oleracea Mart; Acai oil.

\section{Resumen}

La palma de açaí (Euterpe oleracea Mart.) Es una palmera nativa del Amazonas, que se encuentraen grandes extensionesenelestuariodel Amazonas. Durante elproceso de conservación y extraccióndel aceite, factores como lahumedad, la temperatura, las enzimas lipídicas y la presencia de luz puedenafectarel perfil lipídico, 
favoreciendolahidrólisis y laliberación de ácidos grasos. La presencia de ácidos grasosinfluyeenlacalidad de los aceites. Este trabajotuvo como objetivo laextraccióndel aceite de açaí por solvente orgánico y elanálisis de algunosparámetros físico-químicos como: densidad, niveles de acidez y perfil glicídico. Para laextracción se utilizóun aparato soxhlet y hexano como disolvente. La extracción de aceite de açaí no arrojóbuenosrendimientos, debido a laelección de utilizar frutos enteros que tienengrancantidad de fibras envueltasenlassemillas. La densidad se analizó utilizando un densímetro ANTON PAAR 35n, la acidez se midió mediante titulación de KOH y para el perfil glicídico, el aceite se sometió a análisis de EspectroscopíaInfrarroja por Transformada de Fourier (FTIR) utilizando el IR Prestige-21 de Shimadzu. La densidadfueunpoco baja. El nivel de acidez mostró valores ligeramente altos, indicando laformación de ácidos grasos, enel perfil de glicéridos se pudo observar la presencia de ácidos grasos libres, lo que justifica la alta acidez.

Palabras clave: Açaí; Euterpe oleracea Mart; Aceite de acai.

\section{Introdução}

O açaizeiro (Euterpe oleracea Mart.) é uma palmeira nativa da Amazônia, que ocorre em grandes extensões no estuário amazônico (Silva et al., 2019). Em seu conteúdo orgânico, o açaí possui nutrientes interessantes como altos conteúdos de lipídeos $(40,75 \%)$, carboidratos $42,5 \% \pm 3,56$, proteínas $8,13 \mathrm{~g} \pm 0,63$ por $100 \mathrm{~g}$ de açaí liofilizado, além de vitaminas e minerais como cálcio, ferro, magnésio e potássio (Menezes; Torres; Srur, 2008).

Os frutos e sementes de açaí apresentam propriedades funcionais, como a atividade antioxidante, devido à presença de polifenóis, flavonoides e antocianinas. O açaí é rico em ácidos graxos monoinsaturados e poli-insaturados, (Schauss et al., 2006; Nascimento et al., 2008; Rufino et al., 2011) sendo encontrados os ácidos graxos como o ácido linoleico, ácido oleico e ácido palmítico (Menezes; Torres; Srur, 2008). Esses óleos têm interessantes aplicações nutracêuticas, pois o organismo não produz naturalmente.

Durante o processo de extração e armazenamento, os triacilgliceróis podem ser reduzidos a ácidos graxos. A presença de ácidos graxos saturados e insaturados tem relação com a qualidade do óleo de açaí (Perez et al., 2018). A extração convencional do óleo de açaí é realizada com solventes orgânicos em altas temperaturas. Faz-se necessário a realização de estudos da qualidade dos óleos de açaí, obtidos pela extração realizada com solventes orgânicos. O valor elevado do índice de acidez significa que o óleo esta sofrendo hidrólise em suas cadeias, sendo reduzido a ácidos graxos (Azmir et al., 2013; Castro \& a-Ayuso, 1998).

O presente estudo é uma pesquisa de natureza quantitativa (Koche, 2011), tem como finalidade avaliar a extração e caracterização dos parâmetros físico-químicos do óleo de açaí, extraído a partir do fruto inteiro in natura.

\section{Metodologia}

\subsection{Colheita}

A matéria-prima utilizada consiste em frutos de açaí maduros e uniformes que foram colhidos na Fazenda Serra Verde situada na cidade de Curionópolis-PA. Os frutos foram triturados e acondicionados por 3 horas, em estufa a $40^{\circ} \mathrm{C}$ para secagem, ao final, foram pesados, obtendo uma massa de 340 gramas.

\subsection{Obtenção do óleo}

A técnica escolhida para a obtenção do óleo de açaí foi extração por solvente orgânico utilizando-se o aparelho Soxhlet (KA, RV 10 Control, Wilmington, NC, EUA) e o hexano como solvente, de acordo com a metodologia de Santos et al., (2021). Neste método os frutos foram triturados para aumentar o contato de superfície da amostra com solvente. Para obtenção do óleo a amostra foi divida em 3 partes: 100g; 110g e 130g colocadas em cartuchos de papel filtro. A recuperação do hexano foi realizada através da destilação, utilizando um evaporador rotativo. 


\subsection{Caracterização físico-química}

\subsubsection{Determinação de Densidade}

A massa especifica do óleo foi obtida através do densímetro portátil DMA ${ }^{\mathrm{TM}} 35 \mathrm{n}$ da ANTON PAAR, que utiliza o princípio de oscilação por tubo em U. Para determinação da densidade foram utilizados $2 \mathrm{ml}$ de amostra a temperatura de $20^{\circ} \mathrm{C}$ (Martin et al., 2018; Ceron et al., 2018).

\subsection{2 Índice de acidez}

O índice de acidez foi obtido através da titulação com Hidróxido de potássio $(\mathrm{KOH})$, de acordo com a metodologia

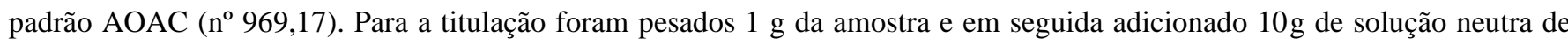
isso-propanol/tolueno, logo após foram adicionados duas gotas de fenolftaleína e titulado com solução alcoólica de KOH, até atingir a coloração rósea.

\subsubsection{Perfil Glicerídico}

As análises de espectroscopia no infravermelho por transformada de Fourier (FTIR) foram realizadas no equipamento IR Prestige-21 da Shimadzu, utilizando uma célula de ATR (Atteunated Total Reflectance) horizontal de $7 \mathrm{~cm}$ de comprimento, analisando a faixa de 600 a $3.500 \mathrm{~cm}-1$ (Siudem et al., 2019).

\subsection{Análise estatística dos resultados}

Os resultados obtidos foram expostos à análise estatística através da Análise de Variância (ANOVA), seguido pelo teste de comparação de médias de Tukey, a 5\% de significância.

\section{Resultados e Discussão}

A extração do óleo de açaí não apresentou bom rendimento, devido à escolha de se utilizar os frutos inteiros, estes apresentaram uma grande quantidade de "fibras" envoltos nas sementes e aparentemente uma quantidade significativa de umidade, o baixo rendimento também pode ser explicado pelos diferentes métodos de extração. O teor lipídico representou 3,55\% da amostra total Tabela 1. Esse teor lipídico é superior aos resultados (2,75\%), reportados por Mello et al., (2021), os quais fizeram a extração do óleo da semente em base seca liofilizada e inferior aos resultados (43,1\%) de Buratto et al., (2021) que realizaram extração do óleo da polpa, pelo método de extração pressurizada assistida por micro-ondas.

Tabela 1: Rendimento da extração do óleo de açaí.

\begin{tabular}{lllllll}
\hline Amostra & Peso inicial $(\mathrm{g})$ & Peso final $(\mathrm{g})$ & Extrato $(\mathrm{g})$ & $\%$ em óleo & Acidez $(\mathrm{KOH} / \mathrm{g})$ & Densidade \\
Fruto & $110 \pm 15,27$ & $79,8 \pm 4,89$ & $4,05 \pm 0,17$ & $3,55 \pm 0,38$ & 0,04 & 0,7645 \\
\hline
\end{tabular}

* 1. Resultados de média \pm desvio padrão $(\mathrm{p}<0,05)$. Fonte: Autores.

Foi observado um índice de acidez ( $0,04 \mathrm{~g}$ de $\mathrm{KOH} / \mathrm{g}$ de amostra) um pouco elevado, conforme Tabela 1 . A acidez dos óleos é uma consequência da hidrólise dos triacilgliceróis que ocorre na semente ou no fruto, em condições de alta umidade, a decomposição é acelerada por aquecimento e pela luz, sendo a rancidez, quase sempre acompanhada pela formação de ácidos graxos livres. O alto valor da acidez pode estar ligado à umidade ou a incidência de luz (Rodrigues et al., 2005; Speranza et al., 2016).

Por meio da análise do espectro FTIR é possível observar a natureza hidrocarbônica da amostra baseado nos estiramentos observados na região de 3.000 a $2.800 \mathrm{~cm}-1$. A pequena banda que pode ser observada em torno de $3.010 \mathrm{~cm}-1$ 
indica a presença de insaturações nas cadeias carbônicas conforme a Figura 1.

Figura 1: analise por infravermelho do óleo de açaí.
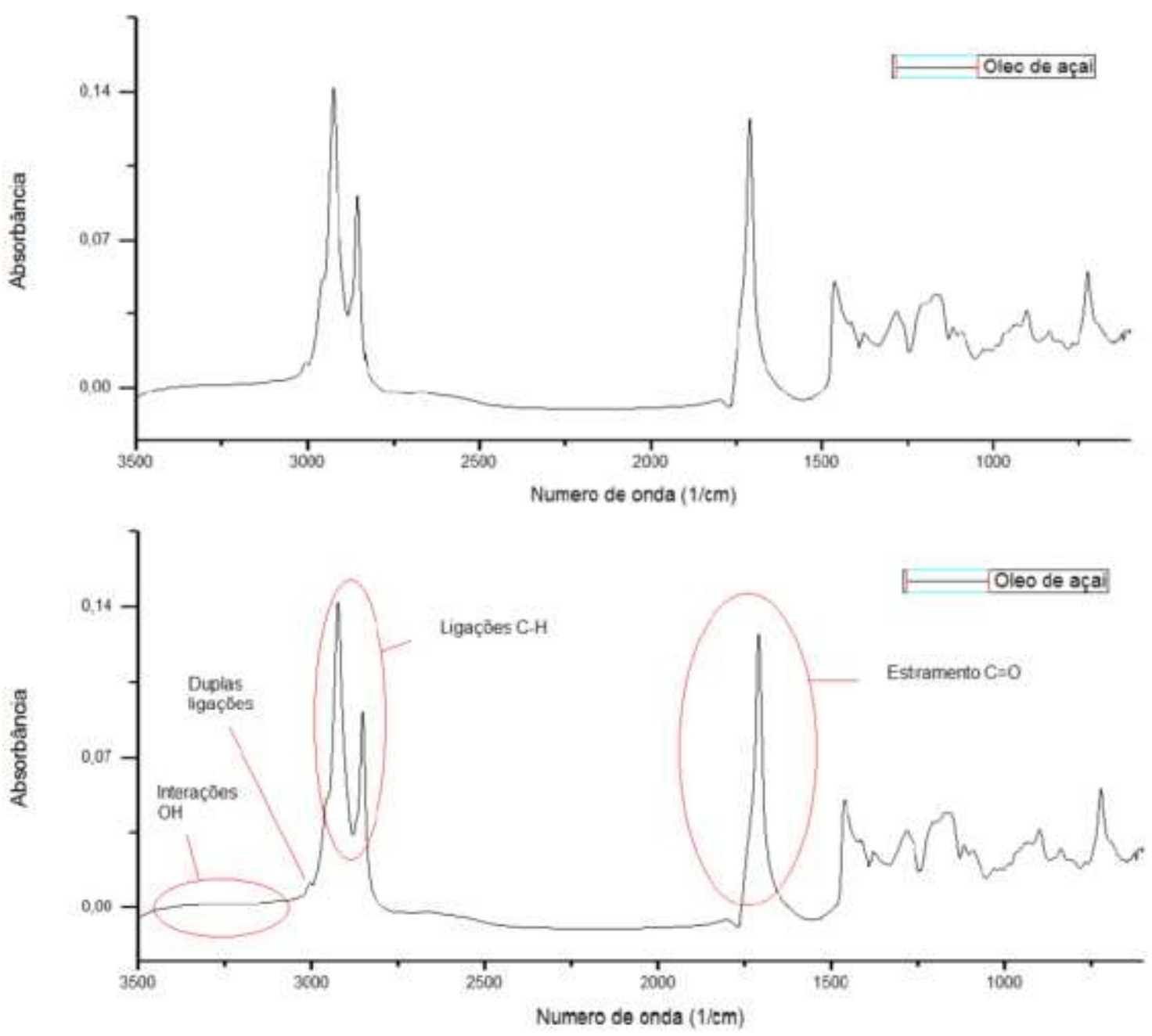

Fonte: Autores.

A caracterização como óleo graxo pode ser observada na banda que se encontra na região de 1.760 a $1.620 \mathrm{~cm}-1$. Esta região é característica do estiramento $\mathrm{C}=\mathrm{O}$, onde a região em torno de $1.740 \mathrm{~cm}-1$ corresponde aos estiramentos $\mathrm{C}=\mathrm{O}$ de grupos éster (Zhang et al., 2021) e $1.710 \mathrm{~cm}-1$ corresponde aos estiramentos $\mathrm{C}=\mathrm{O}$ de grupos ácido carboxílico (Araujo et al., 2021). Observa-se que existe a presença dos dois grupos, ou seja, os ácidos graxos presentes no óleo encontram-se na sua forma glicerídica, entretanto existe a presença de considerável quantidade de ácidos graxos livres, conforme indicado pelo alto valor do índice de acidez do óleo extraído. A região em torno de $3.400 \mathrm{~cm}-1$ corrobora a presença de ácidos graxos livres, uma vez que se observa uma pequena elevação nesta região, que corresponde às interações do tipo ligação de hidrogênio, realizadas por grupos O-H (Buratto et al., 2019).

\section{Conclusão}

O óleo de açaí (Euterpe oleracea Mart) apresentou um baixo rendimento na utilização de frutos inteiros. A acidez mostrou-se levemente elevada, vários podem ser os fatores para estes valores alterados, como à hidrólise enzimática, umidade ou a incidência de luz. A acidez indica a presença de ácidos graxos, comprovado pela análise espectro FTIR.

Os resultados demonstram a importância da escolha da parte do açaí para realizar a extração. Sabemos que o método 
de extração, influência no rendimento do produto, sendo interessante novos estudos com outros métodos de extração.

\section{Referências}

Araujo, R. O., Santos, V. O., Ribeiro, F. C. P., Chaar, J. S., Pereira, A. M., Falcão, N. P. S., \& Souza, L. K. C. (2021) Magnetic acid catalyst produced from acai seeds and red mud for biofuel production. Energy Conversion and Management, 228, 113636. https://doi.org/10.1016/j.enconman.2020.113636

Azmir, J., Zaidul, I. S. M., Rahman, M. M., Sharif, K. M., Mohamed, A., Sahena, F., Jahurul, M. H. A., Ghafoor, K., Norulaini, N.A.N., \& Omar, A. K. M. (2013) Techniques for extraction of bioactive compounds from plant materials: a review.Journal Food Engineering,v. 117, 426-436. https://doi.org/10.1016/j.jfoodeng.2013.01.014

Buratto, R. T., Cocero, M. J., \& Martín, A. (2021) Characterization of industrial açaí pulp residues and valorization by microwave-assisted extraction. Chemical Engineering and Processing - Process Intensification, 160, 108269. https://doi.org/10.1016/j.cep.2020.108269

Castro, M. L. \& Garciá -Ayuso, L. (1998) Soxhlet extraction of solid materials: an outdated technique with a promising innovative future.AnalyticaChimica Acta, 369, 1-10. https://doi.org/10.1016/S0003-2670(98)00233-5

Cerón, A. A., Boas, R. N. V., Biaggio, F. C., \& Castro, H. F. (2018) Synthesis of biolubricant by transesterification of palm kernel oil with simulated fusel oil: Batch and continuous processes. BiomasandBioenergy, 119, 166-172. https://doi.org/10.1016/j.biombioe.2018.09.013

Koche, J. C. (2011). Fundamentos de metodologia científica: teoria da ciência e iniciação à pesquisa. Ed Vozes 122-126

Martin, L. S., Ceron, A., Oliveira, P. C., Zanin, G. M., \& De Castro, H. F. (2018) Different organic components on silica hybrid matrices modulate the lipase inhibition by the glycerol formed in continuous transesterification reactions. Journal of Industrial and Engineering Chemistry, 1-9. https://doi.org/10.1016/j.jiec.2018.01.029

Menezes, E. M. S., Torres, A. T., \& Srur, A. U. S. (2008) Valor nutricional da polpa de açaí (Euterpe oleracea Mart) liofilizada. Acta Amazonica, 38, 311 -316. https://doi.org/10.1590/S0044-59672008000200014

Melo, S. P., Selani, M. M., Gonçalves, R. H., Paulino, J. O., Massarioli, A. D., \& Alencar, S. M. (2021) Açaí seeds: An unexplored agro-industrial residue as a potential source of lipids, fibers, and antioxidant phenolic compounds. Industrial CropsandProducts, 161, 113204. https://doi.org/10.1016/j.indcrop.2020.113204

Nascimento, R. J. S., Couri, S., Antoniassi, R., \& Freitas, S. P. (2008) Composição em ácidos graxos do óleo da polpa de açaí extraído com enzimas e com hexano. Rev. Bras. Frutic. 30498-502. https://doi.org/10.1590/S0100-29452008000200040

Odendaal, A. Y., \& Schauss, A. G. (2014) Potent Antioxidant and Anti-Inflammatory Flavonoids in the Nutrient-Rich Amazonian Palm Fruit, Açaí (Euterpe spp.). Polyphenols in Human Health and Disease, 219-239. https://doi.org/10.1016/b978-0-12-398456-2.00018-9

Official Methods of Analysis of AOAC International, décimaprimeira ed., 1990, 956.

Perez, M. M., Gonçalves, E. C. S, Salgado, J. C. S., Rocha, M. S., Almeida, P. Z., Vici , A. C., infante, J. C., Guisan, J. M., Rocha-Marti , J., Pessela, C. B. \& Polizeli, M. T. M. L. (2018) Production of Omegas-6 and 9 from the Hydrolysis of Açaí and Buriti Oils by Lipase Immobilized on a Hydrophobic Support.Molecules, 23, 3015. https://doi.org/10.3390/ molecules23113015

Rodrigues, C. E. C., Silva, F. A., Marsaioli, A., \& Meirelles, A. J. A. (2005) Deacidification of Brazil nut and macadamia nut oils by solvent extraction: Liquid-liquid equilibrium data at 298.2 K. Journal of Chemical \& Engineering Data, 50, 517-523. https://doi.org/10.1021/je049687j

Rufino, M. S. M., Pérez-Jiménez, J., Arranz, S., Alves, R.E., Brito, E. S., Oliveira, M. S., \& Saura-Calixto, F. (2011) Açaí (Euterpe oleraceae) 'BRS Pará': a tropical fruit source of antioxidant dietary fiber and high antioxidant capacity oil. Food Research International, 44, $2100-2106$. https://doi.org/10.1016/j.foodres.2010.09.011

Santos, A. K., Aguiar, C. M., Silva, E. D., \& Silva, C. (2021) Evaluation of favela seed oil extraction with alternative solvents and pressurized-liquid ethanol. The Journal of Supercritical Fluid, 169, 105125. https://doi.org/10.1016/j.supflu.2020.105125

Schauss, A. G., Wu, X., Prior, R. L., Ou, B., Patel, D., Huang, D., \& Kababick, J. P. (2006) Phytochemical and Nutrient Composition of the Freeze-Dried Amazonian Palm Berry, EuterpeoleraceaeMart. (Acai). Journal of Agricultural and Food Chemistry, 54, 8598-8603. https://doi.org/10.1021/jf060976g

Silva, P. M., Cunha, V. M. B., Sousa, S. H. B., Menezes, E G. O., Bezerra, P. N., Neto, J. T. F., Filho, G. N. R., Araújo, M. E. \& Carvalho JR., R. N. (2019) Supercritical CO2 extraction of lyophilized Açaí (Euterpeoleracea Mart.) pulp oil from three municipalities in the state of Pará, Brazil, Journal of CO2 Utilization, 31, 226-234. https://doi.org/10.1016/j.jcou.2019.03.019

Siudem, P., Wawer, I., \& Paradowska, K. (2019) Rapid evaluation of edible hemp oil quality using NMR and FT-IR spectroscopy. Journal of Molecular Structure, 1177, 204-208. https://doi.org/10.1016/j.molstruc.2018.09.057

Speranza, P., Ribeiro, A. P. B., \& Macedo, G. A. (2016) Application of lipases to regiospecificinteresterification of exotic oils from an Amazonian area. Journal of Biotechnology, 218, 13-20. https://doi.org/10.1016/j.jbiotec.2015.11.025

Zhang, X., Liao, X., Gong, Z., Li, X., \& Jia, C. (2021) Formation of fatty acid methyl ester based microemulsion and removal mechanism of PAHs from contaminated soils. Journal of Hazardous Materials, 413, 125460. https://doi.org/10.1016/j.jhazmat.2021.125460. 